\title{
Discussing the Study of High Tc Properties of Superconductors with Electron Paramagnetic Resonance (EPR) Spectrometers
}

\author{
D. Shaltiel
}

Received: 5 July 2013 / Accepted: 7 August 2013 / Published online: 27 August 2013

(C) Springer Science+Business Media New York 2013

\begin{abstract}
This work discusses investigations of properties of superconductors with the electron spin resonance (ESR) spectrometers. A significant number of articles were published on this subject. Many authors have tried to explain results derived when applying the (ESR) spectrometers to investigate the properties of superconductors. We show that, except for a single publication, most have missed a very important issue, namely, that it is not possible to study superconducting properties with the electron spin resonance spectrometers unless the mechanism that induces the ESR signals is understood. None of the authors were aware that the mechanisms that induce ESR signals in superconductors must be different than the ESR mechanism that induces signals in compounds that holds spins. Explanations that describe this issue are presented.
\end{abstract}

Keywords Electron spin resonance $\cdot$ Electron paramagnetic resonance $\cdot$ Spectrometer

\section{Introduction}

Following the discovery of high Tc superconductors, there has been an influx of publications related to applying conventional Electron Spin Resonance (ESR) spectrometers to investigate the properties of high Tc superconductors. The study was motivated through the observation that when applying the ESR spectrometer, signals were observed. It is an unexpected result as the spectrometer is designed to yield

D. Shaltiel $(\bowtie)$

Racah Institute of Physics, The Hebrew University, Jerusalem, Israel

e-mail: shaltiel@vms.huji.ac.il signals that hold spins. But high Tc superconductors do not hold spins.

Experimental results, obtained by applying the ESR spectrometer to study superconducting properties, are presented in the book: "Superconductors, Properties Technology and Applications," edited by Yuri Evgenievich Grigorashvily. The book was published by Intech Janeza Trdine 9, 51000 Rijeka, Croatia. All chapters are open access. Free online edition of the book is available at [www.Intechopen.com]. It is an interesting and recommended book for scientists interested in the superconductivity field. It contains chapters written by scientists known for their contribution to fundamental research in the superconducting field. Chapters 4 and 5 in Grigorashvily's book present specific results obtained with the ESR spectrometer. They describe the observed signals and how they are formed. The reader is advised to study these two chapters.

Chapter 4 entitled "Investigating superconductivity with Electron Paramagnetic Resonance (EPR) spectrometer" presents ESR results obtained when investigating high temperature superconductors (in superconducting single crystals). The introduction to that chapter states: "The electron paramagnetic spectrometer is a powerful tool to investigate properties of superconductors."

Chapter 5 entitled: "Non Resonance Microwave Absorption (NRMA) Anomalies in High Temperature Superconductors" discusses ESR results in high Tc (superconducting powder samples). The introduction to that chapter states: "High temperature superconductors are known to show some interesting properties, especially magnetic field dependent microwave absorption effects, adding that striking examples are the appearance of very strong nonresonant microwave absorption NRMA signals in the superconducting state, using a conventional electron spin resonance (ESR) spectrometer." As high Tc superconductors 
are not conventional paramagnetic compounds that do not hold spins, signals cannot originate from non-resonant microwave absorption (namely, magnetic dipolar transitions between spin levels). Therefore, when analyzing experimental results derived with an ESR spectrometer, it is necessary to explore the mechanisms that induce signals in the superconductors, including new superconductors that have been discovered recently. New unknown properties of the superconductors may be obtained, provided that the mechanism that induces the ESR signals is understood. Chapter 4 that describes the investigation of superconducting single crystals presents properties of high Tc superconductors that have not been published earlier. To follow how the ESR signals are formed, it is necessary to look for the process that forms the signals. We reemphasize that ESR signals could be induced with the spectrometer only through a resonance process. Therefore, naming ESR results as "non-resonance microwave absorption" (NRMA) is inappropriate, and misleading. Accordingly, a notation different from NRMA has to be applied.

To further explain the measurements using the ESR spectrometer, we repeat that the spectrometer was designed to provide signals in compounds that hold spin. When ESR signals are observed in compounds that do not hold spins, to discover how such signals were obtained, it is necessary to search for the mechanism that can induce the signals. As most scientists are not familiar with the process that forms the ESR signals, we therefore present a description that could help researchers understand the process; the spectrometer was designed to deliver signals in compounds that hold spins. Signals are obtained only when the ACfrequency of the spectrometer is in phase with the frequency of the rotating spins. The AC frequency is adjusted manually, or through the spectrometer.

As the superconducting compounds do not hold spin, we are faced with the important and interesting problem of explaining the process that forms ESR signals in superconductors. Obviously, when such a process is discovered, it would be different than the process that submits signals in compounds that hold spins.

\section{Discussion}

The results presented in Chaps. 4 and 5 discuss the study of high Tc superconductors with the ESR spectrometers. The chemical composition in these two chapters is similar. Different response is obtained when studying either single crystals or powdered material. The different response is related to the structure of the superconductor's single crystals, and to the actual composition of the powder samples. ESR results, obtained by investigating single crystals, can enable determining differences in the interacting intensity between the nearest neighbors of Josephson Vortices. (See Chap. 4 in the book edited by Grigorashvily.) When ESR signals were obtained in powder samples, it was observed that JVs were always present. As Chap. 4 discuses single crystals and Chap. 5 discusses powdered samples, it was observed that investigating single crystals may enable us to determine the interaction between JV nearest neighbors (see Chap. 4). We therefore conclude that ESR signals could be observed only in the presence of JV. Furthermore, it cannot be possible to determine the interaction between specific elements in powder samples. The reason is that nearest neighbor's elements are randomly distributed in powders. Thus when presenting results obtained in powders or in single crystals, it is important to note the differences in the investigated compounds, even if their chemical compositions are similar. We have earlier indicated that the relative position of elements in single crystals can be determined when analyzing the ESR results. Finding the specific position of such elements could present information related to interaction between nearestneighbors elements. In Chap. 5, where powder samples are investigated, the various elements are randomly distributed. Therefore, it is not possible to determine the position of the nearest neighbor elements. Obtaining the relative positions of the nearest neighbors could deliver necessary information in studying the properties of the investigated superconductors.

Presently, we describe how measurements in Chap. 4 or in Chap. 5 were performed. In Chap. 4, the superconducting single crystals were introduced into the spectrometer in a predetermined orientation. Then, the ESR measurements were run at various crystal orientations (relative to the orientation of the ESR magnetic field). In Chap. 5, superconducting powder samples were introduced into the spectrometer, then the ESR measurements were run by applying the regular procedure used to investigate samples that contain spins.

Studies that may lead to obtaining properties of superconductors should be performed by searching for the mechanisms that form the ESR signals. We remark that none of the results of Chap. 5 or in the references presented in this chapter discuss any mechanism that explains how the superconducting ESR signals are obtained. The chapter submits about 65 references related to investigations of superconductors with ESR spectrometers. A study of the results presented in Chap. 5 indicates that neither the authors of Chap. 5 nor the authors of the references presented in Chap. 5 were aware that the investigated superconductors do not hold spins, and that there should be a certain mechanism that is different from the mechanism that submits ESR signals in compounds that contain spins. It is not possible to analyze the ESR result unless one realizes that the investigated superconductors do not hold spins. This point is important for those interested in understanding how ESR signals are formed or how to apply the ESR technique to derive properties in superconductors. 
Experimental results obtained from the measurements presented in Chap. 5 show the following interesting results: (a) ESR signals have been observed only in superconducting samples that hold Josephson Vortices, (b) peak type signals as a function of magnetic field were observed, and (c) hysteresis behavior as a function of magnetic field was also observed. The chapter adds the remark "that explaining the experimental results is far from understanding."

Results derived from measurements presented in Chap. 4 indicate that the ESR signals present interesting results that can be used to study the properties of the investigated superconductors. They may enable one to compare experimental results derived in studying single crystals or powder samples.

The following discusses the differences in the study of high Tc superconductors, presented in Chaps. 4 and 5. It will also emphasize the different approaches when carrying out the measurements.

The results that describe the mechanism that induces ESR signals in superconductors are presented in Chap. 4. They explain the properties derived in superconducting Bi2212 and Bi2223 single crystals. Analyzing the results in these two crystals resolves problems related to the mechanism that induces ESR signals in superconducting compounds that do not hold spins. Figure 11 of Chap. 4 explains, in a straightforward way, how the ESR signals are formed: "The figure shows that the signals are formed through the spectrometer current that induced motion of Josephson Vortices." The ESR results in Chap. 5 relate to superconducting powder samples. Although a large number of experimental results are presented, the approach of the authors does not explain how those ESR signals are obtained and what induces their particular form, such as peak signal, and the anomalous hysteresis signals.

\section{Conclusions}

This work presents a discussion on the study of high Tc superconductor properties with the electron paramagnetic resonance spectrometer. A large number of interesting and important results is presented in Chaps. 4 and 5 of Grigorashvily's book. The experimental results in the two chapters have been published earlier. They do not describe the text and how the results were derived. Instead, the reader is referred to the results that have already been published. This procedure was preferred to avoid repetitions of published results (that explain in detail and clearly how they were obtained). It is not an accepted procedure to present results, as the reader is not able to read them directly, but has to search them using a computer. We repeat that the results were published earlier in a free online edition. The results presented in Chap. 4 solve the most important problem. Chapter 5 explains how signals were derived in superconductors that do not hold spins: "The ESR signals are obtained by inducing motion of Josephson Vortices." The motion of JVs is obtained when the intensity of the AC field is large enough to overcome the penning of JVs. Therefore, studying the ESR signal shape and signal intensities as a function of different parameters, such as magnetic field or temperature, could submit information on the nature of the interaction between Josephson vortices in superconductors, such as those that were discovered earlier or presently. The reader is referred to various figures of Chap. 5 that show results obtained in powder samples. Figure 5 in Chap. 5 presents a peak signal as a function of temperature. The previous conclusions indicate that ESR signals are obtained only if the AC field intensity is strong enough to overcome the pinning of the JVs. As the AC field stays constant during the variation of the temperature, it can be concluded that during that time the JV pinning remains constant. Therefore, the JV density has to increase and decreases sharply during the temperature variation. Or alternatively, the JV pinning strength varies with the temperature. The peak effect could have been studied in detail by applying various procedures, such as field cooling, or by varying the composition of the powder samples systematically. In Chap. 4, where single crystals were investigated, peak signals were also observed. A proper analysis could explain the observed results in the single crystals, and could present additional information of properties in the superconducting compounds. Figure 11 of Chap. 4 describes how the ESR signals have been derived.

Before presenting additional comments (see below), I present a copy of a letter entitled: "Full Chapter Revue." The letter was addressed to me by Professor Yury Grigorashvily. It refers to the manuscript that was published in Chap. 5 of the book "Superconductors, Properties, Technology, and Applications"

"Investigating superconductivity with Electron Paramagnetic Resonance (EPR)

Spectrometer"

Authors: David Shaltiel and Hans-Albrecht Krug Von Nidda Book editor: Yury Grigorashvili

Status: Accepted

Full chapter review

January 04, 2012

Editor's comments:

Dear authors of the manuscript,

I have read your paper titled "Investigating superconductivity with Electron Paramagnetic Resonance (EPR) spectrometer" with great interest. I think that readers of our book will also find it fascinating. Chapter's text is informative, very precise, and logical. I can highlight the goal of this chapter according to your idea written here: "The work shows that an electron paramagnetic resonance spectrometer is a powerful tool to investigate properties of superconductors."

But I can also add that great scientific and applied interest is present in your measured and collected data, 
memory effect, received by studying the monocrystal hightemperature superconductors.

There is also a theoretical analysis of observed effects. I'd like to see more written about it in the conclusion of the chapter. Otherwise, all attention is given to the device as a research instrument only.

I must say that among the peer-reviewed manuscripts, there is one with very similar content. You can see the tentative list of contributing authors (and read the accepted abstracts-"Non resonant microwave absorption (NRMA) Studies in High Temperature Superconductors (HTS)." In this chapter, EPR spectrometer Bruker ER 200D X-band $(9.47 \mathrm{GHz})$ is used and $(\mathrm{Bi}, \mathrm{Pb})-2223$ bulk samples are investigated.

I would like to pay attention to the following parts of your chapter.

As the chapter states, the microwave signal reacts to the 2212 and 2223 crystals' orientation. It also has no response in the YBC compound at any orientation. This is a very important result that has to be brought to readers' attention.

\section{Final Remarks}

Recently, a significant number of new unknown superconductors have been discovered. I draw the attention of the readers that the purpose in applying the ESR spectrometers was to investigate the properties superconductors, including those that have been discovered recently. These works describe the ESR technique. They also show interesting results related to the interaction between the JVs and neighboring elements, including the interaction between the Josephson vortices themselves. Based on accepted theories, the observed results could not have been understood by applying the accepted methods to investigate ESR properties.

Various authors have tried to explain newly published superconducting properties of observed ESR signals without realizing that the superconductors do not hold spins. Therefore, we doubt if their published explanation is correct. A rush to publish interpretations of the newly discovered results, before even understanding the mechanisms that induce such results, is found in various publications. Studying those publications may lead the reader to understand them wrongly. We, the authors of Chap. 4, have noticed that to understand the properties of ESR signals in superconductors it is crucial to discover the mechanism that yields the results. We have spent more than ten years trying to discover the mechanism that induces the ESR signals in superconductors. The conclusion regarding the usage of ESR spectrometers to investigate the properties of superconductors is to use single crystals. Signals derived from powders indicate the presence of JVs. Further studies of the powders may present interesting results which can be used to study the properties of superconductors.

It is suggested that in order to analyze those results, one has to study the powders by introducing more variables in a systematic way, such as additional elements, or applying different cooling processes.

Acknowledgement I would like to thank B. Bogoslavsky for his contribution in this article. 